\title{
Presentación de un modelo de análisis de la conversación y experiencias de aprendizaje mediado en la interacción de sala de clase
}

\author{
Villalta-Paucar, Marco Antonio; Martinic-Valencia, Sergio; Assael-Budnik, Cecilia; Aldunate-Ruff, Nerea \\ Presentación de un modelo de análisis de la conversación y experiencias de aprendizaje mediado en la \\ interacción de sala de clase \\ Revista Educación, vol. 42, núm. 1, 2018 \\ Universidad de Costa Rica, Costa Rica \\ Disponible en: http://www.redalyc.org/articulo.oa?id=44051918008 \\ DOI: https://dx.doi.org/10.15517/revedu.v42i1.23431
}

Esta obra está bajo una Licencia Creative Commons Atribución-NoComercial-SinDerivar 3.0 Internacional. 
Artículos

\title{
Presentación de un modelo de análisis de la conversación y experiencias de aprendizaje mediado en la interacción de sala de clase
}

\author{
Presentation of a Model Analysis of the Conversation and Learning Experiences Mediated in the Classroom \\ Interaction
}

Marco Antonio Villalta-Paucar [1]

Universidad de Santiago de Chile, Chile

DOI: https://dx.doi.org/10.15517/revedu.v42i1.23431

marco.villalta@usach.cl

Sergio Martinic-Valencia [2]

Pontificia Universidad Católica de Chile, Chile

smartini@uc.cl

Recepción: 29 Febrero 2016

Redalyc: http://www.redalyc.org/articulo.oa?id=44051918008

Cecilia Assael-Budnik [3]

Universidad Diego Portales, Chile

cecilia.assael@mail.udp.cl

Nerea Aldunate-Ruff [4]

Universidad de Santiago de Chile, Chile

nerea.aldunate@usach.cl

\section{Notas DE AUTOR}

[1] Psicólogo, Universidad Nacional Mayor de San Marcos. Lima, Perú, Doctor en Ciencias de la Educación, Pontificia Universidad Católica de Chile. En la actualidad es académico e investigador de la Universidad de Santiago de Chile, Facultad de Humanidades, Escuela de Psicología. Director interino de la Escuela de Psicología de la Universidad de Santiago de Chile en el año 2014. Es investigador principal del Proyecto financiado por el Fondo Nacional de Ciencia y Tecnología (FONDECYT) del gobierno de Chile, n. 1150237 titulado: "Experiencias de aprendizaje mediado y análisis de la conversación en la sala de clase de escuelas de diverso contexto socioeducativo" y co-investigador de proyecto Fondecyt a cargo del Dr. Sergio Martinic. En 2015 presenta dos publicaciones en revista científicas: Villalta, M., Guzmán, M. A., y Nussbaum, M. (2015). Procesos pedagógicos y uso de tecnología en el aula. Revista Complutense de Educación, 26(2), 405-424. En http:// dx.doi.org/10.5209/rev_RCED.2015.v26.n2.43303. ISSN: 1130-2496; Martinic, S. y Villalta, M. (2015) La gestión del tiempo en la sala de clases y los rendimientos escolares en escuelas con jornada completa en Chile, Perfiles Educativos, XXXVII(147), 28-49, http://www.iisue.unam.mx/ perfiles/perfiles_articulo.php?clave=2015-147-28-49 y capítulo de libro: Villalta. M. (2015) Processus méthodologique d'analyse de l'interaction didactique en lien avec les connaissances scolaires/la connaissance scolaire. Chapitre 15 (417- 450). En Yves Lenoir et Rocio Esquivel (editores), Les méthodes en usage dans des centres de recherche et chez des chercheurs français et latino-américains. Tome 2. Groupéditions Éditeur, Longueuil, Quebec. ISBN: 978-2-923656-41-0.

[2] Antropólogo, Universidad de Chile; Magister en Ciencias Sociales, FLACSO, Mexico; Phd Sociología Universidad Católica de Louvain, Bélgica. En la actualidad es académico de la Facultad de Educación de la Pontificia Universidad Católica de Chile, Santiago de Chile, Chile. Es investigador principal del Proyecto financiado por el Fondo Nacional de Ciencia y Tecnología (FONDECYT) del gobierno de Chile, n. ${ }^{\circ} 1150931$ titulado: "El aprendizaje de la profesión en contextos reales de trabajo. Estudio comparativo de actividades de prácticas formativas en los casos de medicina y educación" y es co-investigador de Proyecto Fondecyt a cargo del Dr. Marco Villalta. Entre las publicaciones más importantes del 2015 cabe señalar las publicaciones en revistas científicas: Martinic, S. \& Villalta, M. (2015) La gestión del tiempo en la sala de clases y los rendimientos escolares en escuelas con jornada completa en Chile, Perfiles Educativos, XXXVII (147), 28- 49; y, Martinic, S. (2015) El tiempo y el aprendizaje escolar la experiencia de la extensión de la jornada escolar en Chile. Revista Brasileira de Educacao, 2015, 20(61), 479-499.

[3] Educadora Diferencial, Universidad de Chile; Doctora en Ciencias de la Educación con Mención en Interculturalidad, Universidad de Santiago de Chile. En la actualidad es directora del Centro de Desarrollo Cognitivo, Facultad de Educación, Universidad Diego Portales (http:// www.cdc.udp.cl/), Santiago de Chile, Chile. Es co-investigadora del Proyecto financiado por el Fondo Nacional de Ciencia y Tecnología (FONDECYT) del gobierno de Chile, n. 1150237 titulado: "Experiencias de aprendizaje mediado y análisis de la conversación en la sala de clase de escuelas de diverso contexto socioeducativo", a cargo del Dr. Marco Villalta. Entre las publicaciones más importantes de los últimos tres años cabe señalar: Villalta, M.; Martinic, S.; Assael C. (2013) Conocimiento escolar y procesos cognitivos en la interacción didáctica de la sala de clases. Perfiles Educativos, 35(141), 84-96, y Salas, N., González, F. \& Assael, C. (2013) The Contribution of Dynamic Assessment to Promote Inclusive Education and Cognitive Development of Socio-Economically Deprived Children with Learning Disabilities. Transylvanian Journal of Psychology 3(14), 207-222.

[4] Licenciada en Psicología en la Pontificia Universidad Católica de Chile, Ph.D. en Psicología en la Pontificia Universidad Católica de Chile. En la actualidad es académica de la Universidad de Santiago de Chile, Facultad de Humanidades, Escuela de Psicología, Santiago de Chile, Chile. Es coinvestigadora del Proyecto financiado por el Fondo Nacional de Ciencia y Tecnología (FONDECYT) del gobierno de Chile, n. ${ }^{\circ} 1150237$ titulado: "Experiencias de aprendizaje mediado y análisis de la conversación en la sala de clase de escuelas de diverso contexto socioeducativo", a cargo del Dr. Marco Villalta. 


\section{RESUMEN:}

Las evidencias de los últimos años indican que la calidad del diálogo docente-estudiante en el aula es importante en el logro de aprendizajes. Pero aún no se conocen, de modo suficiente, cuáles diálogos son más beneficiosos, ni qué tipo de modelo de análisis es el más pertinente. El objetivo de este artículo [5] es ilustrar un modelo de análisis de la interacción didáctica en el aula que integre criterios de mediación de la teoría de experiencia de aprendizaje mediado (EAM) y las categorías del análisis de la conversación (AC), específicamente los conceptos de intervención e intercambio. Metodológicamente se trata de un estudio cualitativo de análisis de caso instrumental, que consistió en la filmación de tres clases consecutivas de una profesora de cuarto básico de escuela ubicada en sectores sociales vulnerables de la región Metropolitana de Santiago de Chile. El análisis del registro de observación se realizó con las categorías de la EAM y AC. Los resultados indican que los criterios de la EAM cobran precisión al vincular la acción comunicativa del profesorado con la acción comunicativa del estudiantado - trasladando el foco de observación de la intervención al intercambio-, y los intercambios ganan precisión para describir la acción intencionada del personal docente desde el currículo en relación con las acciones que median el aprendizaje autónomo de sus estudiantes. Se demuestra que hay intercambios en el aula que contienen criterios de la teoría EAM, y aportan a comprender la disposición a aprender en estudiantes que viven en contextos sociales vulnerables.

Palabras ClaVE: aprendizaje, enseñanza, proceso cognitivo, análisis del proceso de interacción, proceso de comunicación.

\section{Abstract:}

The evidence of recent years suggests that the quality of teacher-student dialogue in the classroom is important in learning achievement. But it is not yet known sufficiently dialogues which are more beneficial, and what kind of model analysis is the most relevant. The aim of this article is to illustrate an analytical model of the didactic classroom interaction that integrates mediation criteria to the Mediated Learning Experience (MLE) and the categories of the Conversation Analysis (CA) specifically the concepts of intervention and exchange. Methodologically, this is a qualitative analysis of an instrumental case, which involved the shooting of three consecutive classes of a fourth grade teacher of a school located in vulnerable social sectors of the Metropolitan Region of Santiago de Chile. The analysis of the observation records was carried out with the categories of MLE and CA. The results indicate that the criteria of the MLE are more accurate when linking the communicative action of the teacher with the communicative action of the student - moving the observation focus from the intervention to the exchange- and the exchanges become more accurate to describe the intentional action of the teacher from the curriculum in relation to the actions mediating autonomous learning of students. It results that there are classroom exchanges that contain criteria of the EAM theory, and contribute to understanding the predisposition to learn in students living in vulnerable social contexts.

KEYWORDS: learning, cognitive process, interaction process analysis, communication process.

\section{INTRODUCCIÓN}

La calidad de la interacción profesorado-estudiante es importante en la educación (Slavin, 1996), y el diálogo a la base de la acción educativa en el aula (Molina, 2005) donde confluyen los proceso de enseñanza y de aprendizaje. La investigación de los últimos años aporta importantes evidencias sobre cómo se organiza el diálogo en el aula, pero aún no se conoce de modo suficiente cuáles diálogos son más beneficiosos que otros para el aprendizaje, ni respecto a qué tipo de modelo y logro educativo (Howe y Abedin, 2013).

El presente artículo tiene por objetivo ilustrar un modelo de análisis de la interacción didáctica en el aula que integre criterios de mediación de la teoría de experiencia de aprendizaje mediado (EAM) y las categorías del análisis de la conversación (AC). Integrar cognición y cultura es operativamente posible a través de poner en relación dos tradiciones socioculturales de estudio de la interacción en sala de clase: la experiencia del aprendizaje mediado que se articula desde el principio del carácter cultural de la cognición, y el análisis de la conversación, que se articula desde el principio del carácter dialogal de la cultura.

La teoría de la EAM de Reuven Feuerstein explica el proceso de aprendizaje en función del carácter bio-psico-social de la cognición humana, en el marco de la teoría de la actividad sociocultural de Vygotsky (Tzuriel, 2010). La segunda tradición es el AC que explica el proceso de aprendizaje a través de reglas y métodos culturales de construcción del conocimiento escolar (Candela, 2001a; Cubero et al., 2008; Dekker, Elshout-Mohr, y Wood, 2006; Villalta, 2009; Watson, 1992). 
Ambas tradiciones de estudio confluyen en el análisis de la coordinación de acciones entre el proceso de enseñar y el de aprender, así como en el papel de la cultura en la configuración de los significados que orientan la acción en el espacio-tiempo de la sala de clase. El vínculo entre ambas tradiciones de investigación sobre los sucesos educativos en sala de clase ofrece relevantes posibilidades para comprender y precisar la relación entre aprendizaje y enseñanza, conceptos en sí mismos teóricamente complejos y que, puestos en relación, describen diversos tipos de procesos educativos.

En la primera parte se presentan los referentes teóricos e incidencia en el estudio de la interacción en aula de ambas tradiciones; posteriormente, se presenta la propuesta de complementariedad entre experiencia de aprendizaje mediado (EAM) y el análisis de la conversación (AC) así como los procedimiento de análisis de datos; se ejemplifica con registros categorizados de observación y, finalmente, la discusión y conclusiones sobre las posibilidades de análisis que integren cognición y cultura en la observación de la interacción en sala de clase.

\section{UNA PERSPECTIVA SOCIOCULTURAL DEL DESARRollo COgNITIVO: LA EXPERIENCIA DE APRENDIZAJE MEDIADO (EAM)}

En la teoría de la actividad sociocultural de Vygotsky, el lenguaje es herramienta central del pensamiento en tanto acción que apropia y transforma la cultura (Wells, 2004), En esta perspectiva, la cultura es inherente a la constitución de los procesos psicológicos superiores, los cuales se desarrollan con la participación en situaciones sociales específicas (Baquero, 2004). Así, determinadas interacciones comunicativas son evidencia de procesos de apropiación y construcción del conocimiento. En tal sentido, diversos estudios han buscado determinar los nexos entre la teoría sociocultural vygostkiana del desarrollo cognitivo y las teorías del discurso en perspectiva dialogal (Damianova y Sullivan, 2011; Wegerif, Mercer y Dawes, 1999; Wells, 1993).

El habla es considerada herramienta cultural y herramienta psicológica que transforma el pensamiento y acción tanto colectiva como individual (Mercer y Howe, 2012). En tal sentido, el desarrollo cognitivo está vinculado con la acción semiótica en distintos tipos de actividad (Kozulin, 2000). En el contexto escolar, el desarrollo cognitivo se promueve a través de mediaciones semióticas del profesorado en la zona de desarrollo potencial del estudiantado, esto es, aquello que el estudiantado puede realizar con ayuda de otro sujeto, para llegar a realizarlo de modo autónomo.

Con la aproximación sociocultural a la cognición iniciada por Vygotsky se han desarrollado diversas propuestas de análisis de interacciones en el aula vinculadas al aprendizaje y cognición en tanto proceso y no solo en los resultados (Lehesvuori, 2013), un ejemplo de esto es el habla exploratoria del personal docente para promover el desarrollo cognitivo de estudiantes en el aula, que pone de manifiesto mayor conciencia de la tarea (Wegerif et al., 1999); la indagación de estructuras dialogales vinculadas a demanda cognitiva (Wells y Mejia, 2005); y la teoría de modificabilidad cognitiva estructural de Feuerstein (2006), que destaca el rol docente como mediador responsable, afectivo y competente para promover aprendizajes y el desarrollo de estructuras cognitivas en sus estudiantes (Orrú, 2003).

En la perspectiva de Feuerstein (2006), la mediación del aprendizaje refiere a cambios introducidos en la interacción por un mediador humano, docente, quien selecciona, organiza y planifica las acciones de enseñanza para que sean accesibles al receptor -estudiante-, favoreciendo el aprendizaje directo o autónomo. Esta mediación del aprendizaje es lo que Feuerstein articula en la teoría de EAM, que puede definirse como una interacción intencionalmente mediada entre el niño o la niña y los estímulos del entorno para lograr que este sujeto infantil sea sensible al aprendizaje (Kozulin, 2000).

En esta perspectiva, la mediación está a la base del desarrollo humano, y son interacciones entre profesorado y estudiantes ajustadas a ciertos criterios las que logran que estos últimos grupos sean sensibles al aprendizaje, es decir, interacciones que generan modificaciones en la estructura cognitiva. La EAM no refiere al qué, dónde o cuándo de la interacción, sino que refiere a criterios con los cuales se generan condiciones 
para el aprendizaje. Estos criterios que definen la EAM son unos de tipo universal y otros son criterios diferenciadores (Feuerstein, 2006).

Los criterios de mediación (CM) considerados más importantes y universales son tres: 1) Intencionalidad y reciprocidad, donde quien educa transforma el objeto de enseñanza de tal forma que llame la atención del estudiantado, y que este se involucre en la actividad interactiva; 2) Significado, donde el procura dar sentido, valor afectivo a los contenidos de enseñanza y; 3) Trascendencia, donde el profesorado enseña los principios que van más allá de la información o tarea inmediata (Kozulin, 2000; Tzuriel, 2010). Hay otros nueve criterios de mediación que pueden variar según el tipo de tarea o cultura de los individuos: (1) desafío; (2) competencia; (3) compartir; (4) individualización y diferenciación psicológica; (5) pertenencia (6) regulación y autocontrol (7) alternativa optimista; (8) planificación y logro de objetivos; y (9) ser humano como entidad cambiante (Kozulin, 2000; Tébar, 2007). Ver Tabla 1.

TABLA 1

Criterios de la experiencia de aprendizaje mediado (EAM)

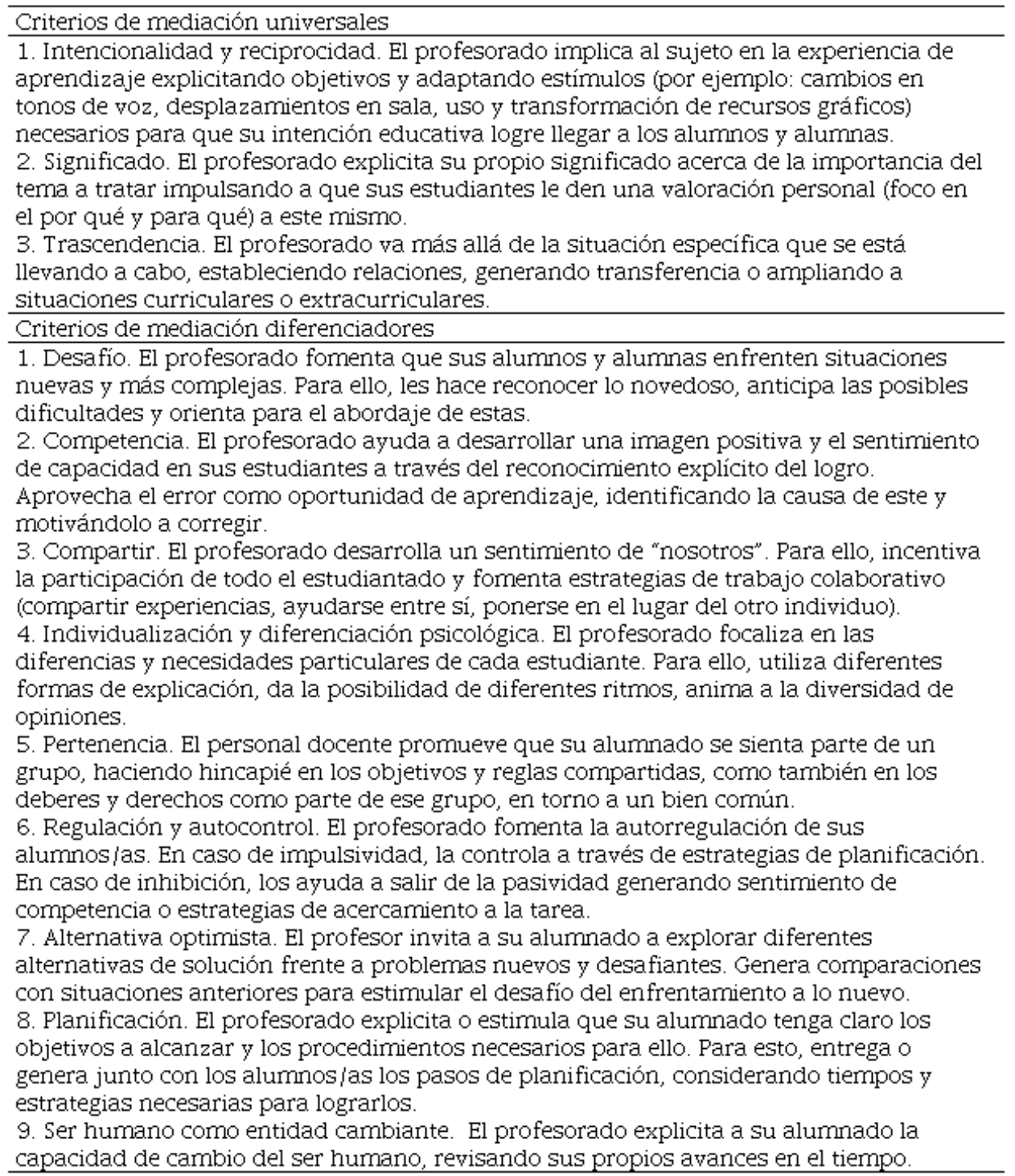


La EAM como teoría y como sistema operacional permite comprender la plasticidad humana y la modificabilidad, además de ser importante referente sobre el tipo de interacción que inciden en el desarrollo humano. La teoría de la EAM tiene la particularidad de haber demostrado acciones educativas con resultados exitosos en niñez y adolescentes en situaciones de alta desventaja social y educativa (Rand, Tannenbaum, y Feuerstein, 1979), poniendo de relieve la acción docente en una propuesta que no pierde la dimensión cultural de la acción del profesorado cuando interviene en los sucesos en sala de clase (Kozulin, 2000; Orrú, 2003).

La perspectiva de Feuerstein se ha formalizado en el Feuerstein Institute (http://www.icelp.info/), y el desarrollo conceptual y operativo de la propuesta se ha estudiado en diversas situaciones educativas (Kozulin et al., 2010); y los CM mencionados se sustentan en los principios de modificabilidad cognitiva estructural y en la teoría de EAM, lo cual fundamenta una metodología de trabajo que integra elementos como la evaluación dinámica de propensión del aprendizaje, el programa de enriquecimiento instrumental (PEI), con los cuales orienta la intervención en el proceso de aprendizaje (Feuerstein, 2015) cuyas etapas han sido cartografiadas en siete pasos que configuran el mapa cognitivo (MC) (Feuerstein, Rand, Hoffman, Egozi, y Shachar-Segev, 2003; Tébar, 2007).

Considerando solamente los CM del complejo entramado conceptual y metodológico del método de Feuerstein, es posible señalar que la EAM: (a) explicita la relación estructural entre cultura y cognición de tal forma que se modifican y transforman recíprocamente; (b) los criterios de mediación consideran de modo amplio la propensión al aprendizaje, en consecuencia no se homologa a contenidos curriculares específicos del aula (Hardman y Delafield, 2010); y (c) el lenguaje verbal es un componente relevante de la mediación (Orrú, 2003) -aunque no se reduce a ello-, y es elemento dominante en la interacción de aula.

Es posible considerar que en el contexto escolar se ponen de manifiesto los CM que definen la EAM, pues la clase está constituida de interacciones comunicativas verbales cargadas de intencionalidad educativa, esto es, actos didácticos sustancialmente semióticos que crean en el receptor la disposición al aprendizaje autónomo (Molina, 2005), y cuyas manifestaciones varían en función de los aspectos socio-histórico-culturales de la escuela.

Se ha estudiado de modo exploratorio la relación entre criterios de la EAM y estructuras comunicativas verbales descritas con el AC en la interacción didáctica de sala, encontrándose asociación entre cierto tipo de estructuras conversacionales y criterios de la EAM (Villalta, Martinic, y Assael, 2013). Esto permite postular que la EAM tiene que ver no solamente con la dimensión ilocutoria de la intervención el profesorado, sino también con los efectos que tiene y la participación de estudiantes en un momento específico de la clase, elementos que son descritos con el análisis de la conversación.

\section{ANÁlisis DE LA CONVERSACIÓN (AC) Y LA CONSTRUCCióN DIALOGAL DEL CONOCIMIENTO ESCOLAR}

El estudio de las propiedades racionales de las acciones contextuales en la vida cotidiana ha sido objeto de estudio de la sociología práctica, que Garfinkel en 1967 denominó como etnometodología (Garfinkel, 2006), para analizar las reglas de construcción de escenarios culturales. Sacks, Schegloff y Jefferson proponen el AC en tanto conjunto organizado de turnos de habla de interlocutores diferentes a lo largo del tiempo (Sacks, Schegloff, y Jefferson, 1974). Ambas perspectivas parten de la base de que la conducta en la vida cotidiana es acción inteligible, y significativa en el contexto de su producción (Pomerantz y Fehr, 2005).

El AC tiene como objeto de estudio la organización secuencial y contextual de la interacción comunicativa para explicar los métodos compartidos que usan los interlocutores para producir y reconocer su propia conducta y la de los demás — sea comunicación verbal y no verbal, en contexto formal o informal(Pomerantz y Fehr, 2005). 
La conversación es el eje articulador que permite conocer cómo se construye y se da sentido a la realidad social (Tusón, 2002), y en su estudio han confluido disciplinas tales como la etnografía de la comunicación, la lingüística interaccional, la filosofía del lenguaje, entre otras (Pomerantz y Fehr, 2005; Tusón, 2002; Villalta, 2009). Conforme se ha incorporado la tecnología de videograbación, el estudio de la conversación es comprendido dentro del campo del análisis de la interacción (Jordan y Henderson, 1995).

El AC aborda al lenguaje en su dimensión interactiva -ilocutora y perlocutoria-, con influencia de la lingüística. Los primeros estudios identificaron las reglas o estructura básica de la conversación profesoradoalumnado en el aula como Inicio-Respuesta-Feedback (IRF) (Candela, 2001b; Kerbrat-Orecchioni, 1998). Dicha estructura básica se confirma y complejiza al describir la cultura escolar en el aula (Villalta, 2009; Wells, 2004). Se ha empleado para estudiar las estrategias docentes para comprometer la participación de estudiantes en las actividades de enseñanza (Nathan y Kim, 2009); para describir las estructuras dialogales que constituyen el conocimiento escolar y conlleva determinadas demandas cognitivas al estudiantado (Villalta, Martinic y Assael, 2013; Wells y Mejia, 2005); describir la interacción ha sido asociada al desarrollo cognitivo (Velasco, 2007), que, en perspectiva de la teoría de la actividad sociocultural, se da a través de la mediación semiótica del profesorado (Wells, 2004).

El AC permite comprender la interacción didáctica como interacción comunicativa especializada de coordinación de acciones para la construcción del espacio intersubjetivo que vincula a los sujetos participantes (Cubero et al., 2008; Marti, 2012) a través de las acciones de enseñar y procesos de aprender conocimientos escolares (Villalta, Martinic, y Guzmán, 2011).

Diversas investigaciones han direccionado el objeto de estudio del AC hacia conocer la construcción del ethos cultural que favorece el aprendizaje escolar, analizando la participación del profesorado en la construcción de diálogos instruccionales (Nathan y Kim, 2009), la cultura colaborativa en la sala de clase (Wells y Mejia, 2005); las tensiones entre participación del estudiantado para aprender, y la participación del profesorado para enseñar (Emanuelsson y Sahlström, 2008), o la relación entre los tipos de intercambio comunicativo y la demanda cognitiva al estudiantado en el aula (Villalta y Martinic, 2013). El estudio de las estructuras de conversación entre docente y estudiante sugiere que la denominada enseñanza efectiva - aquella que se da en aulas de alto aprendizaje escolar-se ajusta a las realidades culturales educativas configurando distintos tipos de rutinas de interacción (Martinic y Villalta, 2015; Pehmer, Gröschner y Seidel, 2015).

En la actualidad se reconocen diversos métodos de AC que buscan revelar las interpretaciones y prácticas que son importantes para los participantes (Pomerantz y Fehr, 2005). Específicamente para el contexto escolar se han descrito las secuencias de tiempo de la clase: Inicio, desarrollo y cierre cuya estructuración depende de la cultura institucional (Martinic y Villalta, 2015). A lo largo de estas secuencias de tiempo se construyen las unidades temáticas o actividades típicas del aula (ATA) que son la expresión de coordinaciones dialogales del curriculo escolar en el aula, las cuales están constituidas por Intercambios, considerada la unidad dialogal mínima de dos interlocutores diferentes -docente y estudiantes- que coordinan sus intervenciones individuales en función de la demanda cognitiva que constituye al conocimiento escolar (Villalta y Martinic, 2013; Villalta et al., 2013).

Desde la perspectiva del AC, los resultados de aprendizaje escolar son expresión de lo afortunado o desafortunado de la coordinación de acciones verbales y no verbales puestos de manifiesto en la interacción en el aula. No obstante, la determinación cualitativa o cuantitativa de estructuras conversacionales asociadas a contextos escolares de diferentes resultados de rendimiento escolar es importante, pero aún insuficiente para inferir aprendizajes, o delimitarlos a la expresión discursiva (Candela, 2001b; Rampton, Roberts, Leung y Harris, 2002). Es necesario profundizar en el vínculo entre lenguaje - en tanto construcción cultural - y cognición - en tanto proceso individual—; aspecto que es abordado por corrientes de la psicología, especialmente la socio-histórico-cultural (Alexander, 2007; Grossen, 2010). 


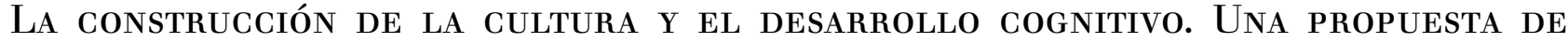 ANÁLISIS DE DATOS DE OBSERVACIÓN DE SALA DE CLASE}

Los estudios sobre el análisis de la interacción didáctica en sala de clase en la perspectiva del AC confluyen en que: (1) hay niveles jerárquicos del lenguaje dialogal: Dialogal -interacción de dos sujetos interlocutores diferentes-, y Monologal —interacción de un sujeto interlocutor consigo mismo-; (2) la conversación se organiza de modo diferente de acuerdo con la gestión del tiempo de la clase; (3) la cultura escolar incide en la definición de estructuras conversacionales; y (4) que el currículo escolar que lleva el profesorado al aula incide en el tipo de demanda cognitiva (Candela, 2001b; Martinic y Villalta, 2015; Villalta, 2009; Villalta et al., 2013).

Es posible establecer relaciones de complementariedad entre los criterios de mediación de la teoría EAM y el AC para el estudio de la interacción en sala de clase. La teoría de experiencia de aprendizaje mediado describe, a través de los 12 criterios de mediación -universales y diferenciadores- las acciones y eventos que impulsa el profesorado y son predisponentes favorables al aprendizaje por parte de sus estudiantes, y el análisis de la conversación describe las condiciones contextuales de secuencias de tiempo y objetivos curriculares -fases e unidades temáticas-y de estructuras conversacionales -intercambios e intervencionesque constituyen la clase. En tal sentido, el vínculo entre AC y EAM aporta a explicar la relación entre proceso y resultado de aprendizaje escolar en la sala de clase (Figura 1).

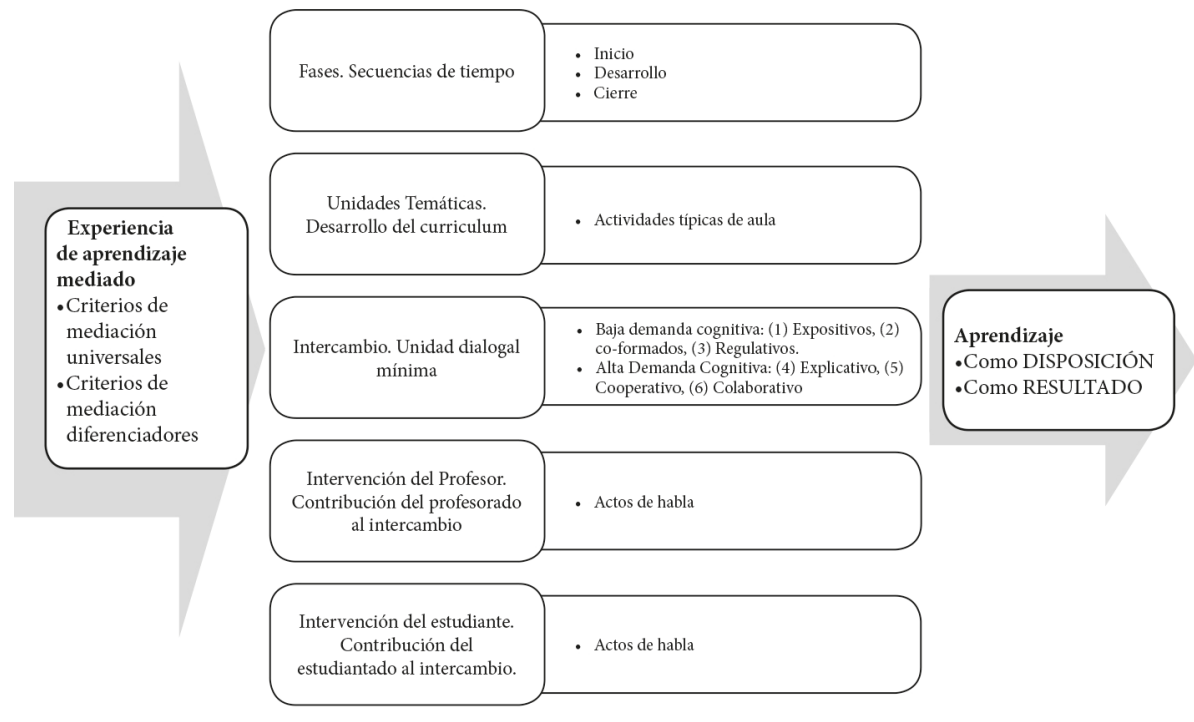

FIGURA 1

Experiencia de aprendizaje mediado (EAM) y análisis de la conversación $(\mathrm{AC})$ en la Interacción didáctica en la sala de clase Elaboración propia

Los criterios de EAM son 12, tres de ellos son criterios de mediación universales, es decir, considerados comunes a toda la existencia humana, independiente de diferencias culturales, socioeconómicas o educativas; y 9 son criterios de mediación diferenciadores, cuya aparición depende de la diversidad de los estilos cognitivos, aspectos culturales y del comportamiento emocional estudiantil. Estos se pueden definir operativamente desde algunos estudios que han generado datos empíricos de clase con los criterios EAM.

Por otra parte, y de modo complementario, el AC aquí propuesto describe la clase en una estructura jerárquica y de secuencia temporal que en un nivel más amplio refiere a la organización global del tiempo de la clase, que se describe en fases: 1) Inicio, 2) Desarrollo, y; 3) Cierre, la cual tiene relación con aspectos amplios de la cultura y rendimiento escolar (Martinic y Vergara, 2007; Martinic y Villalta, 2015). 
En el tiempo que dura la clase se desarrollan unidades temáticas, es decir conjunto de participaciones interactivas con fuerte coherencia semántica y pragmática (Kerbrat-Orecchioni, 1998).

[Estas] ... desarrollan el currículum en el aula, a nivel: a) Declarativo, referido a conocimientos factuales y conceptuales que constituyen una determinada disciplina escolar; b) Procedimental, referido a la aplicación de conceptos o técnicas a una determinada situación planteada y; c) Metacognitivo, referido a la conciencia del propio conocimiento y las condiciones para generarlo. (Villalta et al., 2013, p. 89)

Las unidades temáticas se configuran con intercambios, la mínima unidad conversacional que pone en interdependencia a dos interlocutores diferentes. Se han definido intercambios regulativos o espacio-tiempo (Marti, 2012), que describen la construcción de espacio conversacional en el aula, e instruccionales, que desarrollan los contenidos de la clase (Villalta, 2009), identificándose intercambios de alta y baja demanda cognitiva (Martinic y Villalta, 2015; Villalta et al., 2013) que coordinan determinadas operaciones mentales con el conocimiento escolar (Anderson et al., 2001).

Las estructuras de intercambio descritas según las demandas cognitivas del profesor a sus estudiantes son de seis tipos (Villalta et al., 2013) y es necesario considerar dos precisiones al respecto: (1) los intercambios son la unidad de análisis privilegiada de la interacción comunicativa, los elementos más amplios (fases y unidades temáticas) y más específicos (intervenciones y actos de habla) aportan a comprender el proceso de construcción del intercambio; y, (2) los intercambios son categorías empíricas, sustentadas en la observación de contextos específicos; en consecuencia, su definición y delimitación descriptiva se tiene que adaptar constantemente a cada situación de observación (Tabla 2).

TABLA 2

Intercambios relacionados con la demanda cognitiva

\begin{tabular}{l}
\hline Baja demanda cognitiva \\
1) Expositivo, que describe comunicación unidireccional del profesorado hacia el \\
alumnado. El alumnado tiene comportamiento pasivo de recepción de información. \\
2) Co-formados, docente evalúa dominios dados en clase. El alumnado tiene \\
comportamiento reactivo a la demanda de contenidos que hace su docente. \\
3) Regulativos, docente organiza turnos de habla en la sala. El alumnado tiene \\
comportamiento reactivo ajustado al orden que docente establece en la clase. \\
Alta demanda cognitiva \\
4) Explicativo, alumnado toma iniciativa para obtener información de contenidos. \\
Profesor ado reelabora e incrementa información de contenidos. \\
5) Cooperativo, alumnado asume participación activa en la co-construcción con su \\
docente o compañeros las, de los contenidos de la clase \\
6) Colaborativos, donde el alumnado asume objetivos y modo autónomo de resolución \\
de problemas colaborativamente.
\end{tabular}

Nota: Elaboración propia.

Los intercambios están constituidos por intervenciones consideradas como turnos o acciones comunicativas mutuamente orientadas y aceptadas. Han sido descritas intervenciones del profesorado que favorecen el aprendizaje (Cuadrado y Fernández, 2008); organizadas según el tipo de la demanda cognitiva Alta o Baja desde la intervención del profesorado (Tornero, Ramaciotti, Truffello y Valenzuela, 2015; Wells y Mejía, 2005); a la vez que se ha analizado la interdependencia con la intervención del alumnado (Nathan y Kim, 2009).

La intervención es equivalente al acto de habla en tanto gramática del lenguaje, pero considerada como función interactiva (Kerbrat-Orecchioni, 1998). Descrita comúnmente con un verbo performativo, la unidad privilegiada de estudio ha sido de tipo verbal, no obstante, también se ha considerado el papel comunicativo de los signos no verbales, como por ejemplo, el comportamiento visual en la interacción, el 
cual se releva como un importante mecanismo de regulación del flujo de la conversación (Vertegaal, Slagter, Veer, y Nijholt, 2001).

Se han realizado estudios donde se analiza la asociación entre los criterios de la EAM y las categorías de AC en clases filmadas en educación media (Villalta et al., 2013) y educación básica (Assael, 2015), ambos estudios en establecimientos públicos de la Región Metropolitana de Santiago de Chile. En dichos estudios se encuentra que los criterios de la EAM tienen asociación más fuerte con las Intervenciones del profesorado y con los Intercambios.

Cognición y aprendizaje están estrechamente vinculados por los soportes que ofrece la cultura, especialmente en el contexto educativo de la sala de clase (Schwartz, 2009). Es en el espacio y tiempo de la clase donde se evidencia la eficacia de enseñanza para el logro de aprendizajes en estudiantes (Slavin, 1996); donde elpersonal docente desarrolla determinadas estructuras conversacionales, las cuales son pertinentes al contexto cultural escolar (Villalta et al., 2011); e inciden en el desarrollo cognitivo del estudiantado, especialmente en los primeros años de escolarización (Oliver, 2011). En efecto, la mediación de la cultura que hace el profesorado es determinante en el desarrollo cognitivo de los niños y las niñas (Feuerstein, 2006).

Es de considerar que la EAM y el AC en el contexto de aula aportan complementariamente a comprender el aprendizaje como un proceso constituido de acciones intencionadas por parte del profesorado, coordinada con estudiantes para generar la disposición a aprender y, en consecuencia, inciden en el logro de resultados de aprendizaje escolar.

\section{Metodología}

Se analiza el caso de una profesora con estudiantes de cuarto básico - niñez entre 9 y 10 años- de escuela (a la cual denominamos SIH) ubicada en sector vulnerable de la Región Metropolitana. La escuela seleccionada es considerada como "Autónoma" según la ley de Subvención Escolar Preferencias (Ley SEP), esto significa que sistemáticamente en los últimos cuatro años dicha escuela ha demostrado buenos resultados educativos en la prueba nacional del Sistema de Medición de Calidad de la Educación (SIMCE) chileno en $4^{\circ}$ y $8^{\circ}$ año de educación general básica.

Se filmaron de modo consecutivo tres clases de la asignatura de Lenguaje, para asegurar la habituación de niñez y profesoras a los instrumentos de registro. El registro de información se hizo a través de una cámara filmadora de posición fija en la parte posterior de la sala frente a la profesora, para registrar de modo privilegiado la interacción de ella con los niños y niñas.

La profesora, sus estudiantes, personal directivo y apoderados fueron previamente informados del estudio y se solicitó autorización para registrar información a través de las cartas de consentimiento a las profesoras, de autorización de los padres y madres y asentimiento informado de estudiantes.

El análisis de los registros de información se hizo segmentando la filmación en unidades de 5 segundos, los cuales fueron categorizados según la presencia o ausencia de EAM (descrito en Tabla 1) y de INTERCAMBIOS (descrito Tabla 2). A través de observar reiteradamente las filmaciones se alcanzó acuerdo entre sujetos observadores diferentes para las categorías de EAM y de INTERCAMBIO de ambas clases (Tabla 3). 


\section{Resultados}

TABLA 3

Índice Kappa de concordancia entre observadores de categorías intercambio y EAM

\begin{tabular}{lllll}
\hline $\mathrm{n}$ & CATEGORÍAS & $\mathrm{n}\left({ }^{*}\right)$ & $\begin{array}{l}\text { Índice } \\
\text { Kappa }\left({ }^{*}\right)\end{array}$ & Valoración $\left({ }^{* *}\right)$ \\
\hline 1 & INTERCAMBIO & 586 & 1,00 & Casi Perfecta \\
2 & CRITERIOS DE EXPERIENCIA DE & 181 & 0,61 & Considerable \\
\hline
\end{tabular}

Nota: $\left({ }^{*}\right)$ unidades de tiempo de 5 segundos; $\left({ }^{* *}\right)$ p $<.001 ;\left({ }^{* * *}\right)$ Según Landis \& Koch, 1977. Elaboración propia.

A continuación, se presentan ejemplos de segmentos de trascripción en los que hubo acuerdo de cumplimiento de criterios de EAM. Por razones de espacio para el presente artículo, se presentan solamente los criterios EAM universales observados.

Ejemplo 1: Intencionalidad y reciprocidad.

profesora: <¿la semana pasada?> hicimos un montón de creaciones, montón de textos ahora yo necesito que sepamos ¿ qué tipo de texto?! Cómo dijeron cartas...

(Clase 2, profesora SIH, 2014).

Ejemplo 2: Significado

<profesora pasa por los grupos revisando sus avances. Se detiene en un grupo de niños que tiene problemas para organizar sus roles para realizar el trabajo $>$

profesora: <hay un diálogo previo no audible entre los niños y de ellos con la profesora, se dirige a los niños del grupo> no es que tu leas una pregunta, tu lees otra y tu lees otra, no, <ique dijimos?> compartimos ideas, trabajamos juntos, compartimos ideas

(Clase 2, profesora SIH, 2014).

Ejemplo 3: Trascendencia.

profesora: Cuando hay un conflicto lo tenemos que conversar, no nos enojamos, si no lo conversamos,

(Clase 2, profesora SIH, 2014).

Cabe señalar que no se observaron todos los criterios EAM específicos. En el presente caso analizado se observaron las siguientes EAM diferenciadoras: Competencia, Individuación, Regulación, Desafío, Compartir, Pertenencia y Planificación. No se observa los criterios de EAM diferenciadores: Alternativa optimista y ser humano como entidad cambiante.

Esto es teóricamente aceptable dado que las EAM diferenciadoras tienen relación con aspectos contextuales tales como: la edad, la cultura escolar, el tipo de contenido, entre otros elementos, que inciden en la configuración de formas específicas de interacción.

\section{EAM EN INTERCAMBIOS: UNA RELACIÓN COMPLEMENTARIA.}

A continuación, se presentan las trascripciones de criterios EAM pero esta vez dentro del Intercambio en que fueron clasificados. Igual que en el caso anterior, se ejemplifican solamente los criterios de EAM universales. Se consideran los mismos ejemplos de criterio de mediación puestos esta vez en relación con el INTERCAMBIO. Asimismo, se describe al intercambio por las funciones que cumplen las INTERVENCIONES que la componen: $\mathrm{i}=$ Intervención de Inicio; $\mathrm{r}=$ Intervención de respuesta; $\mathrm{e}=$ 
Intervención de evaluación; e/i=Intervención de evaluación e inicio; y e/c= Intervención de evaluación y cierre

TABLA 4

EAM Intencionalidad en Intercambio co-formado

\begin{tabular}{|c|c|c|c|c|}
\hline $\mathrm{n}$ & Texto (tiempo: 00:01:10 - 00:02:00) & EAM & $\begin{array}{l}\text { Función de } \\
\text { la } \\
\text { intervención }\end{array}$ & Intercambio \\
\hline 1 & $\begin{array}{l}\text { <profesora presenta la actividad de la } \\
\text { clase> profesora: < ¿la semana } \\
\text { pasada?> hicimos un montón de } \\
\text { creaciones, montón de textos ahora yo } \\
\text { necesito que sepamos ¿¿que tipo de } \\
\text { texto?! como dijeron cartas }\end{array}$ & $\begin{array}{l}\text { Intencionalidad } \\
\text { y reciprocidad }\end{array}$ & 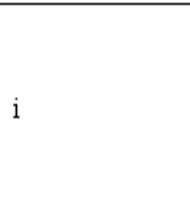 & \\
\hline 2 & $\begin{array}{l}\text { alumnos <levantan la mano antes de } \\
\text { hablar > jafiches! }\end{array}$ & & $r$ & \\
\hline 3 & $\begin{array}{l}\text { profesora: 〈repite aceptando la } \\
\text { respuesta, y con los brazos va dando la } \\
\text { palabra a los niños〉 afiches }\end{array}$ & Regulación & $e / i$ & Co formado \\
\hline 4 & alumna: ¡comics! & & $\mathrm{r}$ & \\
\hline 5 & profesora: comics & Competencia & e & \\
\hline 6 & alumna: jinvitación! & & $\mathrm{r}$ & \\
\hline 7 & profesora: invitación & Competencia & e & \\
\hline 8 & alumno: recetas & & $\mathrm{r}$ & \\
\hline 9 & $\begin{array}{l}\text { profesora recetas, ¿eso lo hicieron } \\
\text { ustedes aquí? }\end{array}$ & Competencia & eji & \\
\hline 10 & alumnos (a coro) si:.: & & $\mathrm{r}$ & \\
\hline 11 & profesora: bien & Competencia & $\mathrm{e} / \mathrm{c}$ & \\
\hline
\end{tabular}

Nota: Elaboración propia.

Se observa (Tabla 4) que la intervención n. ${ }^{\circ} 1$ inicia el intercambio co-formado con criterio EAM de intencionalidad y reciprocidad, pero también las intervenciones 3 a 9 describen un proceso dialogal mediado por la intervención con criterio EAM diferenciadora de regulación y competencia; la intervención n. ${ }^{\circ} 11$, que cierra el intercambio, simultáneamente refuerza positivamente la participación del estudiantado (EAM diferenciadora Competencia). 
TABLA 5

EAM Significado en intercambio cooperativo

\begin{tabular}{|c|c|c|c|c|}
\hline n. ${ }^{\circ}$ & Texto (tiempo: 00:31:15 - 00:32:30) & EAM & $\begin{array}{l}\text { Función de la } \\
\text { intervención }\end{array}$ & Intercambio \\
\hline \multirow[b]{2}{*}{1} & $\begin{array}{l}<\text { profesora pasa por los grupos revisando sus } \\
\text { avances. Se detiene en un grupo de niños que } \\
\text { tiene problemas para organizar sus roles para } \\
\text { realizar el trabajo> }\end{array}$ & & & \multirow{6}{*}{ Cooperativo } \\
\hline & $\begin{array}{l}\text { profesora: < hay un diálogo previo no audible } \\
\text { entre los niños y de ellos con la profesora, se } \\
\text { dirige a los niños del grupo> no es que tu leas } \\
\text { una pregunta, tu lees otra y tu lees otra, no, } \\
<\text { ¿que dijimos?> compartimos ideas, trabajamos } \\
\text { juntos, compartimos ideas }\end{array}$ & Significado & $\mathrm{i}$ & \\
\hline 2 & alumno: <¿entonces cerramos los libros?> & & $\mathrm{r}$ & \\
\hline 3 & $\begin{array}{l}\text { profesora: ¡no! no se trata de cerrar todo, alguien } \\
\text { que lea la pregunta, <todos dan su respuesta?> } \\
\text { alumnos: (mostrando el cuadernillo de trabajo }\end{array}$ & Planificación & e/i & \\
\hline 4 & $\begin{array}{l}\text { entregado) < ¿y lo escribimos aquí la respuesta } \\
\text { de cada uno?> }\end{array}$ & & $\mathbf{r}$ & \\
\hline 5 & $\begin{array}{l}\text { profesora: claro, <puedes escribir, Miguel } \\
\text { respondióXXX?> (lo dice mientras se aleja } \\
\text { del grupo) }\end{array}$ & Regulación & $\mathrm{e} / \mathrm{c}$ & \\
\hline
\end{tabular}

Nota: Elaboración propia.

En la Tabla 5 el intercambio cooperativo se inicia con intervención de profesora ajustada a criterio universal EAM de significado, intervención 3 introduce la EAM diferenciadora planificación y cierra con intervención que simultáneamente contiene criterio EAM diferenciadora regulación. 
TABLA 6

EAM Trascendencia en intercambio colaborativo

\begin{tabular}{|c|c|c|c|c|}
\hline n. ${ }^{\circ}$ & Texto (tiempo: 01:17:45 - 01:20:20) & EAM & $\begin{array}{l}\text { Función de la } \\
\text { intervención }\end{array}$ & Intercambio \\
\hline 1 & $\begin{array}{l}\text { Al final de la clase, donde los niños han realizado } \\
\text { una prueba entre dos, la profesora pregunta } \\
\text { cuales son las cosas a favor y contra de que los } \\
\text { niños puedan resolver una prueba entre dos. Se } \\
\text { presenta e segmento donde indaga las razones } \\
\text { en contra de hacer una prueba entre dos: }\end{array}$ & & $\mathrm{i}$ & \\
\hline & profesora: ¿y cuáles serian las en contra? & & & \\
\hline 2 & $\begin{array}{l}\text { profesora: / estamos pensando primero. Un } \\
\text { minuto para pensar, no te veo pensar < se dirige } \\
\text { a niño } 4>\text {. }\end{array}$ & Regulación & I & \\
\hline 3 & $<$ silencio de 5 segundos aproximadamente $>$ & & & \\
\hline 4 & niño 5: que el compañero no estudia & & $\mathrm{r}$ & \\
\hline 5 & $\begin{array}{l}\text { profesora: el compañero no estudia }<\text { da la } \\
\text { palabra a niño } 4>\end{array}$ & & $\mathrm{e}$ & \\
\hline 6 & $\begin{array}{l}\text { niño } 4 \text { : el compañero no puede ser amigable y } \\
\text { molesta mucho }\end{array}$ & & $\mathrm{r}$ & \\
\hline 7 & $\begin{array}{l}\text { profesora: que moleste mucho, no amigable }<\text { da } \\
\text { la palabra a otro niño }>\end{array}$ & & $\mathrm{e}$ & \\
\hline 8 & $\begin{array}{l}\text { niño 6: que las opiniones son distintas y produce } \\
\text { un clonfic, clonflimto < tiene problemas con la } \\
\text { pronunciación de la palabra }>/\end{array}$ & & $\mathrm{r}$ & Colaborativo \\
\hline 9 & $\begin{array}{l}\text { profesora: /puede producir un conflicto. Al haber } \\
\text { conflictos sabemos cómo resolverlos ¿cierto?, los, } \\
\text { los tenemos que...< <eja espacio para que niños } \\
\text { completen la oración>, que ... }\end{array}$ & Trascendencia & $\mathrm{e} / \mathrm{i}$ & \\
\hline 10 & niño 6: & & $\mathrm{r}$ & \\
\hline 11 & profesora: resolver, ¿cómo lo resolvemos? & Desafío & $\mathrm{e} / \mathrm{i}$ & \\
\hline 12 & niño 7: hablando & & $\mathrm{r}$ & \\
\hline 13 & profesora: hablando. Muy bien. & Competencia & e & \\
\hline 14 & niño 5: no pegándose & & $\mathrm{r}$ & \\
\hline 15 & profesora: no pegándose. Muy bien & Competencia & $\mathrm{e}$ & \\
\hline 16 & $\begin{array}{l}\text { profesora: Cuando hay un conflicto lo tenemos } \\
\text { que conversar, no nos enojamos, si no lo } \\
\text { conversamos. }\end{array}$ & Trascendencia & $\mathrm{e}$ & \\
\hline 17 & $\begin{array}{l}\text { Profesora: Muy bien < da la palabra a otro niño> } \\
\text { conversando se puede resolver }\end{array}$ & & $\mathrm{e}$ & \\
\hline 18 & niños a coro: resolver & & $\mathrm{e} / \mathrm{c}$ & \\
\hline
\end{tabular}

Nota: Elaboración propia en base a (clase 2, 4to Básico, SIH, Lenguaje 2014)

En tanto, en la Tabla 6 se observa que el intercambio colaborativo contiene el criterio EAM universal trascendencia en las intervenciones 9 y 16, más intervenciones ajustadas a criterios EAM diferenciadoras de regulación, desafío y competencia

En síntesis, las estructuras de intercambio en los tres ejemplos contienen y coordinan varios criterios de mediación en un mismo intercambio y los criterios de la EAM cobran mayor precisión perlocutoria al ampliar el campo de observación desde la intervención de la profesora hacia el Intercambio. 


\section{DiSCUSIÓN Y CONCLUSIONES}

Tanto el análisis de la conversación como la teoría de experiencia de aprendizaje mediado comparten el valor que otorgan al lenguaje y contexto sociocultural para comprender los procesos de enseñanza y del aprendizaje. El lenguaje es herramienta cultural de signos que cobran sentido en la comunicación. El estudio de los signos verbales permite la construcción de la unidad de análisis en la conversación y el reconocimiento de la mediación de aprendizaje que hace el profesorado.

El presente estudio pone en evidencia que EAM y AC se complementan en la tarea de describir la coordinación de la acción de enseñar y las acciones de aprender. Los criterios de mediación se enriquecen y cobran mayor precisión empírica de su carácter "mediador" del aprendizaje autónomo al ampliar el análisis de la intervención del profesor hacia el análisis del intercambio docente-estudiante. Por su parte, el AC vincula y precisa la descripción de las estructuras dialogales que constituyen la cultura escolar en el aula -donde se plasma el currículo- con el carácter cognitivo que el proceso dialogal del aula hace posible.

La conversación es la unidad mínima del ethos cultural donde quien educa promueve el aprendizaje y, en consecuencia, la apropiación autónoma del estudiantado de su propia experiencia a través de la modificación de sus estructuras cognitivas. El análisis de la conversación y la teoría de experiencia de aprendizaje mediado (EAM) se complementan para delimitar el estudio de la interacción didáctica de aula en tanto punto de encuentro entre los procesos de aprendizaje y de enseñanza en un contexto sociocultural determinado.

En el presente estudio se trabajó con escuelas de sector social vulnerable y alto rendimiento escolar; y en tal sentido, los resultados presentados desde el modelo de análisis aplicado aportan a la comprensión de los procesos semióticos que constituyen aspectos cotidianos de la sala de clase y la cultura escolar, los cuales las educadoras promueven para favorecer la disposición a aprender en sus estudiantes, y convierten la escuela es un espacio de resiliencia al fracaso educativo.

Por otra parte, la conversación se enriquece con los signos comunicativos que ofrece el contexto -como diversidad social y la cultura escolar, la gestión del tiempo y actividades de la clase- y el comportamiento no verbal de los sujetos interlocutores, especialmente la mirada. Futuros estudios deben integrar los elementos del contexto y de la mirada al AC y a la descripción de la EAM, para dar cuenta de la diversidad cognitiva presente en el aula y la experiencia profesional del personal docente para gestionar el proceso educativo en la sala de clase.

\section{REFERENCIAS}

Alexander, P. (2007). Bridging Cognition and Socioculturalism Within Conceptual Change Research: Unnecessary Foray or Unachievable Feat? [Tender un puente sobre cognición y socioculturalismo dentro de la investigación del cambio conceptual: ¿̇ncursión innecesaria o proeza inalcanzable?] Educational Psychologist, 42 (1), 67-73. doi: https://doi.org/10.1080/00461520709336919

Anderson, L., Krathwohl, D., Airasian, P., Cruikshank, K., Mayer, R., Pintrich, P., Raths, J y Wittrock, M. (2001). A Taxonomy for Learning, Teaching and Assessing: A revision of Bloom's taxonomy of educational objetives. [Una taxonomía para el aprendizaje, enseñanza y evaluación: Una revisión de la taxonomía de objetivos educativos de Bloom] Nueva York: Longman.

Assael, C. (2015). La construcción del otro desde los discursos e interacciones de docentes de eduación básica, trabajando en aulas regulares con diversidad cognitiva y diversidad étnica. Búsqueda de conexiones entre la denominada educación Inclusiva y la educación intercultural (Tesis de doctorado inédita). Universidad de Santiago de Chile, Santiago, Chile.

Baquero, R. (2004). Vigotsky y el aprendizaje escolar. Buenos Aires, Argentina: Aique Grupo Editor.

Candela, A. (2001a). Corrientes teóricas sobre el discurso en el aula. Revista Mexicana de Investigación Educativa, 6(12), 317-333. 
Candela, A. (2001b). Corrientes teóricas sobre el discurso en el aula. Revista Mexicana de Investigación Educativa (RMIE), 6(12), 317-333.

Cuadrado, I., y Fernández, I. (2008). ¿Cómo intervienen maestros y profesores para favorecer el aprendizaje en secundaria? Un estudio comparativo desde el análisis del discurso. Infancia y Aprendizaje, 31(1), 3-23. doi: https://doi.org/10.1174/021037008783487101

Cubero, R., Cubero, M., Santamaría, A., Mata, M. L., Ignacio, M. J., y Prados, M. M. (2008). La educación a través de su discurso. Prácticas educativas y construcción discursiva del conocimiento en el aula. Revista de Educación, 346, 71-104.

Damianova, M. K., and Sullivan, G. B. (2011). Rereading Vygotsky's theses on types of internalization and verbal mediation. Review of General Psychology, 15(4), 344-350. doi: https://doi.org/10.1037/a0025627

Dekker, R., Elshout-Mohr, M., and Wood, T. (2006). How Children Regulate their Own Collaborative Learning. Educational Studies in Mathematics, 62(1), 57-79. doi: https://doi.org/10.1007/s10649-006-1688-4

Emanuelsson, J., and Sahlström, F. (2008). The Price of Participation: Teacher control versus student participation in classroom interaction. Scandinavian Journal of Educational Research, 52(2), 205-223. doi: https:// doi.org/10.1080/00313830801915853

Feuerstein, R. (2006). Instrumental Enrichment. [Enriquecimiento instrumental] Jesuralem, Israel: ICEL, Publications.

Feuerstein, R. (2015). Página institucional de Feuerstein Institute: Overview. Recuperado de http://www.icelp.info/ feuerstein-method/overview.aspx

Feuerstein, R., Rand, Y., Hoffman, M., Egozi, M., and Shachar-Segev, N. (2003). Instrumental Enrichment Program [Programa de Enriquecimiento Instrumental] En R. Feuerstein (Ed.). Feuerstein's Theory and Applied Systems: A Reader (pp.99-128) Jerusalén, Israel: ICELP Press.

Garfinkel, H. (2006). Estudies Estudios en Etnometodología (1 ed.). México: Anthropos Editorial.

Grossen, M. (2010). Interaction analysis and psychology: a dialogical perspective. Integr Psychol Behav Sci, 44(1), 1-22. doi: https://doi.org/10.1007/s12124-009-9108-9

Hardman, M. and Delafield, B. (2010). Philosophy for Children as dialogic teaching. [Filosofía para niños como enseñanza dialógica] In K. Littleton \& C. Howe (Eds.), Educational Dialogues. Understanding and promoting productive interaction (pp. 149-164). London: Routledge.

Howe, C. and Abedin, M. (2013). Classroom dialogue: a systematicreview across four decades of research. Cambridge Journal of Education, 43(3), 325-356. doi: https://doi.org/10.1080/0305764X.2013.786024

Jordan, B., and Henderson, A. (1995). Interaction Analysis: Foundations and Practice. The Journal of the Learning Sciences, 41(1), 39-103. Doi: https://doi.org/10.1207/s15327809jls0401_2

Kerbrat-Orecchioni, C. (1998). Les interactions verbales. Approche interactionnelle et structure des conversations. Tome I. Troisiéme Èdition. [Las interacciones verbales. Aproximación interaccional y estructural a la conversación] Paris: Armand Colin.

Kozulin, A. (2000). Instrumentos psicológicos: la educación desde una perspectiva sociocultural. Barcelona, España: Paidós.

Kozulin, A., Lebeer, J., Madella-Noja, A., Gonzalez, F., Jeffrey, I., Rosenthal, N., and Koslowsky, M. (2010). Cognitive modifiability of children with developmental disabilities: A multicentre study using Feuerstein's Instrumental Enrichment-Basic program. Research in Developmental Disabilities, 31(2), 551-559. doi: https:// doi.org/10.1016/j.ridd.2009.12.001

Lehesvuori, S. (2013). Towards Dialogic Teaching in Science. Challenging Classroom Realities through Teacher Education. (Tesis de Doctorado), University of Jyväskylä, Jyväskylä, Finland.

Marti, L. (2012). Tangential floor in a classroom setting. System, 40(3), 398-406. doi: https://doi.org/10.1016/ j.system.2012.07.006 
Martinic, S., y Vergara, C. (2007). Gestión del tiempo e interacción del profesor-alumno en la sala de clases de establecimientos con jornada escolar completa en Chile. REICE - Revista Electrónica Iberoamericana sobre Calidad, Eficacia y Cambio en Educación, 5(5), 3-20.

Martinic, S., y Villalta, M. (2015). La gestión del tiempo en la sala de clases y los rendimientos escolares en escuelas con Jornada Escolar Completa en Chile. Perfiles Educativos, 37(147), 28-49. doi: https://doi.org/10.1016/ j.pe.2013.03.001

Mercer, N., and Howe, C. (2012). Explaining the dialogic processes of teaching and learning: The value and potential of sociocultural theory. Learning, Culture and Social Interaction, 1(1), 12-21. doi: https://doi.org/10.1016/ j.lcsi.2012.03.001

Molina, S. (2005). Acto Didáctico y lenguaje: perspectiva neuropsicológica. Enseñanza, 23, 287-304.

Nathan, M. J. and Kim, S. (2009). Regulation of Teacher Elicitations in the Mathematics Classroom. Cognition and Instruction, 27(2), 91-120. doi: https://doi.org/10.1080/07370000902797304

Oliver, M. (2011). Towards an understanding of neuroscience for science educators. Studies in Science Education, 47(2), 211-235. doi: https://doi.org/10.1080/03057267.2011.604478

Orrú, S. E. (2003). Reuven Feuerstein y la Teoría de la Modificabilidad Cognitiva Estructural. Revista de Educación, $332,33-54$.

Pehmer, A.-K., Gröschner, A., and Seidel, T. (2015). How teacher professional development regarding classroom dialogue affects students' higher-order learning. Teaching and Teacher Education, 47, 108-119. doi: https:// doi.org/10.1016/j.tate.2014.12.007

Pomerantz, A., y Fehr, B. (2005). Análisis de la conversación: enfoque de estudio de la acción social como prácticas de producción de sentido. In T.v. Dijk (Ed.), El discurso como interacción social (pp. 101-139). Barcelona: Gedisa.

Rampton, B., Roberts, C., Leung, C., and Harris, R. (2002). Methodology in the Analiysis of Classroom Discourse. Applied Linguistics, 23(3), 373-392. doi: https://doi.org/10.1093/applin/23.3.373

Rand, Y. A., Tannenbaum, A. J., and Feuerstein, R. (1979). Effetcs of Instrumental Enrichment on the Psychoeducational Development of Low-Functioning Adolescents. Journal of Educational Psychology, 71(6), 751-763. Doi: https://doi.org/10.1037/0022-0663.71.6.751

Sacks, H., Schegloff, E., and Jefferson, G. (1974). A Simplest Systematics for the Organization of Turn-Taking for Conversation. Languaje, 50(1), 696-735. Recuperado de http://www.cs.columbia.edu/ julia/cs4706/ Sacks_et_al_1974.pdf

Schwartz, M. (2009). Cognitive Development and Learning: Analyzing the Building of Skills in Classrooms. Mind, brain, and education, 3(4), 198-2008. Doi: https://doi.org/10.1111/j.1751-228X.2009.01070.x

Slavin, R. E. (1996). Salas de Clase Efectivas, Escuelas Efectivas: Plataforma de Investigación para una Reforma Educativa en América Latina PREAL. Recuperado de http://bit.ly/2BB79iS

Tébar, L. (2007). El profesor mediador del aprendizaje (1 ed.). Santiago de Chile: Arrayan Editores.

Tusón, A. (2002). El análisis de la conversación: entre la estructura y el sentido. Estudios de Sociolingüística, 3(1), 133-153.

Tornero, B., Ramaciotti, A., Truffello, A., y Valenzuela, F. (2015). Nivel cognitivo de las preguntas que formulan las educadoras de párvulos. Educación y Educadores, 18(2), 261-283. doi: https://doi.org/10.5294/ edu.2015.18.2.5

Tzuriel, D. (2010). Programa de Intervención basado en la propuesta de aprendizaje mediado. En Centro de Desarrollo Congnitivo de la Universidad Diego Portales (Ed.). Seminario Internacional "Familias y Jardín Infantil: Juntos aseguran el futuro" Serie de Reflexiones Infancia y Adolescencia $\mathrm{N}^{\circ}$ 12. (pp.79-93). Santiago, Chile: UNICEF. Recuperado en http://www.unicef.cl/web/wp-content/uploads/doc_wp/WD\%2012-Hogar \%20de\%20Cristo.pdf

Velasco, A. (2007). Un sistema para el análisis de la interacción en el aula. Revista Iberoamericana de Educación, 42(3), $1-12$. 
Vertegaal, R., Slagter, R., Veer, G. v. d., and Nijholt, A. (2001). Eye Gaze Patterns in Conversations: There is More to Conversational Agents Than Meets the Eyes. Actas de la Conferencia de SIGCHI en Factores Humanos en Sistemas de Computación (pp.301-308) Seattle, Washington, USA. doi: https:// doi.org/10.1145/365024.365119

Villalta, M. (2009). Análisis de la conversación. Una propuesta para el estudio de la interacción didáctica en sala de clase. Estudios Pedagógicos, 35(1), 221-238. doi: https://doi.org/10.4067/S0718-07052009000100013

Villalta, M., y Martinic, S. (2013). Interacción Didáctica y procesos cognitivos. Una aproximación desde la práctica y discurso docente. Universitas Psychologica, 12(1), 221-233. Recuperado de http://revistas.javeriana.edu.co/ index.php/revPsycho/article/viewFile/1325/3976

Villalta, M., Martinic, S., y Assael, C. (2013). Conocimiento escolar y procesos cognitivos en la interacción didáctica de sala de clase. Perfiles Educativos, 35(141), 84-96. doi: https://doi.org/10.1016/S0185-2698(13)71836-1

Villalta, M., Martinic, S., y Guzmán, M. A. (2011). Elementos de la interacción didáctica en la sala de clase que contribuyen al aprendizaje en contexto social vulnerable. Revista Mexicana de Investigación Educativa, 16(51), 1137-1158.

Watson, R. (1992). Ethnomethodology, Conversation Analysis and Education: An Overview. Internatiional Review od Education, 38(3), 257-274. doi: https://doi.org/10.1007/BF01101432

Wegerif, R., Mercer, N., and Dawes, L. (1999). From social interaction to individual reasoning: an empirical investigation of a possible socio-cultural model of cognitive development. Learning and Instruction, 9(6), 493-516. doi: https://doi.org/10.1016/S0959-4752(99)00013-4

Wells, G. (1993). Reevaluating the IRF Sequence: A Proposal for the Articulation of Theories of Activity and Discourse for the Analysis of Teaching and Learning in the Classroom. Linguistics and Education, 5, 1-37. doi: https://doi.org/10.1016/S0898-5898(05)80001-4

Wells, G. (2004). Dialogic Inquiry. Towards a Sociocultural Practice and Theory of Education. [Investigación dialógica. Hacia una práctica sociocultural y Teoría de la Educación] Toronto: Cambridge University Press.

Wells, G., and Mejia, R. (2005). Toward dialogue in the classroom: learning and teaching through inquiry. [Hacia el diálogo en el aula: aprendizaje y enseñanza a través de la investigación] Working Papers on Culture, Education and Human Development 1(4), 1-45. Recuperado de https://rei.iteso.mx/bitstream/handle/11117/3009/Wells\%2c\%20G.\%2c\%20\%26\%20Mej \%c3\%ada-Arauz\%2c\%20R.\%20\%282005\%29.\%20Toward\%20dialogue\%20in\%20the\%20classroom.pdf? sequence $=2$

\section{Notas}

[5] Auspiciado por Fondo Nacional de Ciencia y Tecnología (FONDECYT) del Gobierno de Chile, Proyecto n. ${ }^{\circ}: 1150237$ titulado "Experiencias de aprendizaje mediado y análisis de la conversación en la sala de clase de escuelas de diverso contexto socioeducativo".

\section{BY-NC-ND}

